\title{
Surface-brightness profiles of dwarf galaxies in the NGC 5044 Group: Implications for the luminosity-shape and scalelength-shape relationships as distance indicators
}

\author{
C. K. Young ${ }^{1,2,3,4}$ and M. J. Currie ${ }^{5}$ \\ 1 Shanghai Astronomical Observatory, Chinese Academy of Sciences, 80 Nandan Road, Xuhui District, \\ Shanghai 200030, PR China \\ 2 National Astronomical Observatories, Chinese Academy of Sciences, 20A Datun Road, Chaoyang District, \\ Beijing 100012, PR China \\ 3 Department of Physics, Hong Kong University, Pokfulam Road, Hong Kong, China \\ 4 Department of Astrophysics and Optics, School of Physics, the University of New South Wales, Sydney 2052, \\ Australia \\ 5 Joint Astronomy Centre, 660 North A'ohoku Place, Hilo, Hawaii 96720, USA
}

Received 6 January 2000 / Accepted 14 January 2000

\begin{abstract}
In a recent paper, which presents CCD photometry for fifteen dwarf and intermediate early-type galaxies in the NGC 5044 Group, it has been claimed that "a few relatively bright galaxies with "convex" profiles destroy the known relation between total magnitude and the "shape" parameter... thus ruling out the use of this relation as a distance indicator for individual galaxies". In the same paper, further reasons were cited supposedly "limiting also its use as a distance indicator for groups of galaxies". We demonstrate that none of the three relatively bright galaxies cited as possessing "convex" profiles actually has a convex profile, and that one of these objects should be excluded because it is a late-type galaxy. Of the two remaining objects, one has an anomalous profile shape whilst the other is brighter than one might expect from its colour alone. However, we show that all of the other issues raised have already been accounted for by Young \& Currie (1994, 1995 \& 1998). The main implications of the new observations are: (1) that the case of one galaxy with an anomalous profile shape, N42, highlights the need for some a priori criteria to be defined in order to establish objectively which objects are not suitable for distance determinations; and (2) on the basis of another unusual galaxy, N50, colour has now been shown to be a poorer discriminant between objects of the same profile shape and scalelength (but of different central surface brightness) than previously thought. How significant this latter problem is depends on how common N50-like objects are. This consideration reinforces the case for always using the more general scalelength-shape relationship of Young \& Currie (1995) in preference to the luminosity-shape one of Young \& Currie (1994). Reassuringly, through a re-analysis of the same CCD photometry, we find that NGC 5044 Group galaxies observe a tight scalelength-shape relationship. This finding supports the view that the scalelength-shape relationship is a viable distance indicator.
\end{abstract}

Key words. galaxies: structure - galaxies: photometry - galaxies: fundamental parameters - galaxies: elliptical and lenticular, cD - galaxies: distances and redshifts - galaxies: clusters: individual: NGC 5044 Group

\section{Introduction}

Based on new surface photometry for fifteen dwarf and intermediate luminosity elliptical galaxies in the NGC 5044 Group, Cellone (1999) reached the conclusions that:

(1) "the shape-luminosity relation cannot be used to derive distances to individual objects, at least until the situation of bright dwarfs with convex profiles, like N42 and N50, is understood";
(2) "its use to derive mean relative distances for groups of galaxies is severely hampered by the fact that its slope changes when objects of different intrinsic effective radii are compared"; and

(3) "the scatter of the $L-n$ relation remains relatively high" even if the three outliers are eliminated.

The purpose of the present paper is to address all of these issues.

Send offprint requests to: C. K. Young,

e-mail: cky@bat.phys.unsw.edu.au 


\section{The three outliers}

The three objects that allegedly destroy the luminosityshape correlation are N42, N49 and N50. Let us now deal with each object in turn.

The claim that N42 has a convex profile was based on a fit only to a short section of this object's luminosity profile, ignoring both the innermost regions corresponding to mean radial distances (hereunder $r^{*}$ ) of less than $18^{\prime \prime}$, and the outermost regions beyond $r^{*} \sim 47^{\prime \prime}$. As Cellone himself mentions, the very substantial luminosity excess in the inner regions cannot be due to a semi-stellar nucleus alone. If it were merely the product of a semi-stellar nucleus and seeing effects, its luminosity profile should have a $F W H M$ of about 2 ". 4 , corresponding to the azimuthally averaged mean seeing disc at the time of observation. In fact, the inner profile has a $F W H M$ of 5 !' $^{\prime}$, which means that the intrinsic luminosity distribution must itself have a FWHM of 4." 4. This demonstrates the presence of a core. In Young \& Currie (1994), we emphasized that whilst nuclei should not (for obvious reasons) be modelled, cores should be. If one now fits to the whole profile out to 60 "' 0 , ignoring only the innermost $r^{*}<2$ "' 0 , i.e. the region most distorted by seeing effects, one finds that the profile is markedly concave, with an $n$ value of 0.60 instead of the quoted value of 1.43 . Note that although our new fit does not account for a small luminosity excess between $r^{*} \sim 20^{\prime \prime}$ and $42^{\prime \prime}$, the light lost this way is a minute fraction of that lost by Cellone's fit, as shown in Fig. 1. Once the core of this galaxy is taken into account then, the measured photometric structural parameters change radically.

From Fig. 1, it is clear that Sérsic's (1968) profile parameterisation does not trace precisely the radial light distribution of N42, although it is already known to be a good approximation to the global profile shapes of most dwarf ellipticals and dwarf spheroidals. The luminosity profiles of atypical objects (such as tidally truncated ones and/or those containing large quantities of dust, undergoing star formation and/or merging) clearly cannot be expected to conform to the same scaling laws as those observed by typical dwarf ellipticals. When measuring distances it is therefore both necessary and justifiable to exclude those objects that may be tidally truncated by massive neighbours or already known to possess colour gradients. Certainly, this galaxy's luminosity profile appears to be anomalous, and may well be tidally truncated as noted by Cellone. Alternatively, significant colour gradients may be the cause, but further observations would be needed in order to establish this. We accept that it would be better to decide which objects in a galaxy sample are suitable for distance measurement on the basis of objective a priori information rather than simply rejecting objects whose profiles are not well fitted by Sérsic's law. As in Young \& Currie (1998) we therefore recommend that colour-gradient information be obtained for all sample galaxies in advance of attempting to measure distances from profile shapes.

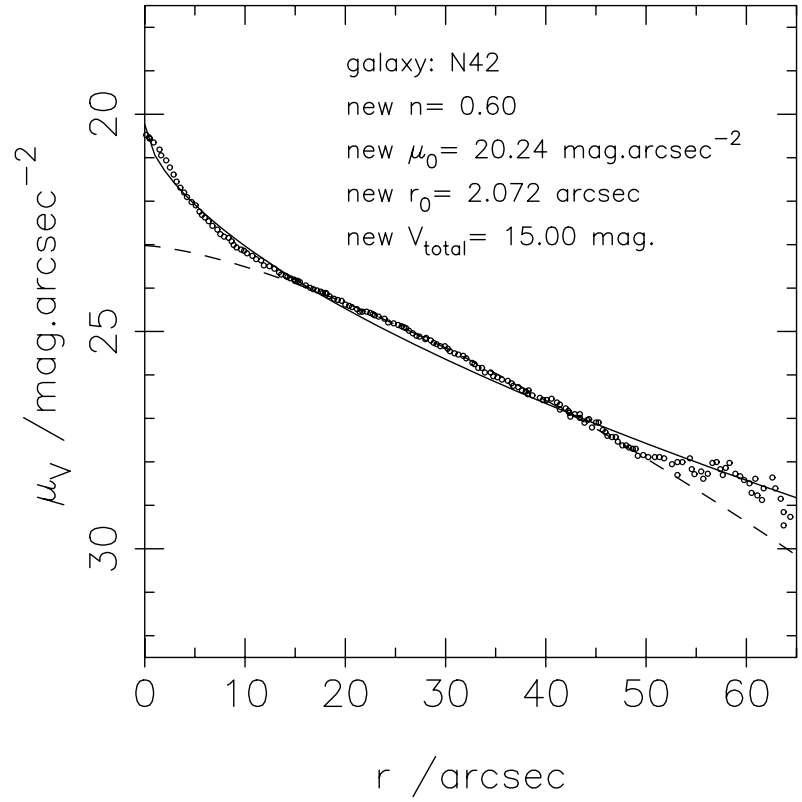

Fig. 1. Cellone's $V$-band surface-brightness profile of N42. His fit (dashed line) and our new fit (solid line) are both shown. Although this profile exhibits some departures from a purely Sérsic one, note the relative sizes (if converted to linear intensity units) of the luminosity excesses not taken into account in each case. For reference, we have listed our new values for N42's Sérsic profile parameters: $n$ ( $N$ in Cellone's notation), $\mu_{0}\left(S_{0}\right), r_{0}(\alpha)$; and our new value for $V$-band total magnitude: $V_{\text {total }}\left(V_{\mathrm{T}}\right)$. The data points were obtained by digitising Cellone's Fig. 1

As far as N49 is concerned, Cellone himself agrees that this object must have a different stellar population from the majority of his sample galaxies on account of its very blue colour of $B-V=0.49$, which is often taken as evidence for recent star formation. As we cautioned many times in Young \& Currie (1994, 1995 \& 1998), objects with different stellar populations are not comparable using the $L-n$ correlation, though they are using the more general $R-n$ correlation. N49's relatively high luminosity with respect to most of the sample galaxies is therefore not anomalous. In fact, it would be more surprising if it did obey the same $L-n$ correlation as defined by redder sample galaxies. Moreover, even Cellone concedes that this galaxy may not be a dwarf elliptical at all, but an irregular of type ImIII as typed by Ferguson \& Sandage (1990). Assuming this is the case, then this object simply should not be in his dwarf-elliptical galaxy sample at all.

This leaves N50, which is curious in that it has a colour typical of ordinary dwarf ellipticals, $B-V=0.76$, but has a higher than normal apparent luminosity. That this galaxy might be a foreground object has already been ruled out by Cellone's preliminary velocity measurement for it, so there is a strong case for this object being of higher than normal intrinsic luminosity too. Let us now investigate the origin of this high luminosity. From Cellone's profile parameters for N49 and N50, it is clear that both of these galaxies have almost identical profile shapes and are 
also of almost identical apparent size. This means that, if they are at essentially the same distance, which seems most likely, they must both obey the same $R-n$ relationship. This is some reassurance at least and demonstrates that the difference in intrinsic luminosity is a function only of a surface brightness shift that is essentially invariant with respect to $r^{*}$.

\section{Distances to individual galaxies}

As we noted in Young \& Currie (1995), colour is not a monotonic function of $M / L$, central surface brightness, or stellar population. More specifically, our conclusions at the time were, as re-iterated in Young \& Currie (1998): "the $L-n$ relationship appears to be most useful for those dwarf ellipticals with colours of $(B-V) \approx 0.7$, because other dwarfs are generally bluer... regardless of whether they are higher or lower surface-brightness objects (YC95)". It was for these reasons that we only applied the $L-n$ relationship to a colour-restricted subset of our Virgo galaxy sample.

As Cellone cited Durrell et al.'s (1996) finding that the $L$ - $n$ relationship supposedly yielded an unreliable distance in one case; it is worth mentioning here that Durrell et al. were comparing objects of very different colours (and therefore stellar populations too). As with N49, it would indeed be surprising if Durrell et al.'s objects did obey the same $L-n$ relationship as one another.

Cellone's discovery of a high surface brightness dwarf elliptical of $(B-V)=0.76$ is, however, quite significant. It now shows that even in the most useful colour range, colour cannot be a fail-safe discriminator between objects of the same size and profile shape but of different central surface brightness. In practical terms, this means that when the $L-n$ relationship is applied to individual galaxies of $(B-V) \sim 0.7$ without taking into account differences in central surface brightness, a small minority of objects may be assigned spurious distances. How serious this problem is does of course depend on how common these anomalously bright objects really are. As taking into account central surface brightness in work based on the $L-n$ relationship is equivalent to working with the more general $R$-n relationship for which objects like N50 do not pose any problem, it does now appear that one might as well use this latter relationship from the outset.

We would also like to take this opportunity to reemphasize that contrary to the misinterpretation of Young \& Currie (1995) advanced by Binggeli \& Jerjen (1998) and repeated by Cellone, our intention was never to use profile shapes to measure large numbers of high-accuracy distances to individual galaxies. Our intention was always to measure distances to ensembles of galaxies based on large numbers of approximate distances to individual objects.

In many cases galaxy ensembles are obvious groups or clusters. However, it is already known that a high proportion of apparent clusters comprise more than one component (e.g. the Centaurus Cluster, whose double structure was revealed in velocity space by Lucey et al. 1986; and even the Coma Cluster, whose complex structure is reviewed by Biviano 1997). In Young \& Currie (1995) we felt that it was appropriate to look statistically at a histogram of galaxy counts as a function of individual galaxy distance estimates in the Virgo direction, where the presence of distinct groups approximately in the same line-of-sight might explain the large scatter observed in various scaling laws, including those based on the profileshape parameter. In fact, we will soon be presenting a very much expanded study of substructure in the spatial distribution of galaxies in the Virgo direction. This new study will have several major improvements over Young \& Currie (1995), including: a very much larger galaxy sample, more accurate distance measurements based on higher resolution photometry and a large number of new radialvelocity measurements.

\section{Mean distances to groups or clusters}

Unless objects analogous to N50 are at some later date shown to be quite common, the discovery of one such object does not pose any major problem to the application of the $L-n$ relationship to whole groups or clusters of galaxies. However, Cellone raised an additional issue, namely that the slope of the $L-n$ relationship varies with $n$, and that "this requires that samples" [of galaxies in different groups but] "with the same distribution of intrinsic sizes must be observed, a goal that is usually not easy to achieve". Such a criticism could of course be levelled at virtually any other distance indicator in that one must compare like objects at different distances; rather than e.g. giant ellipticals in distant clusters with dwarf ellipticals in nearby ones, simply because distant dwarfs are hard to observe. Few if any, scaling laws can be linearly extrapolated with confidence and the $L-n$ relationship is no exception. The obvious answer is to observe objects in each group over a wide enough range in $L$ and $n$ so as to ensure as much overlap as possible between the two samples.

We do concede that in Young \& Currie (1994) we did not model seeing effects (partly because the seeing disc $F W H M$ for the Fornax photometry was a mere $0{ }^{\prime \prime} 6$ ) and used straight-line fits. However, by Young \& Currie (1995) we were already using polynomial fits and backing this up with a comprehensive treatment of seeing effects. Cellone's point about objects of small angular sizes being unusable (and this affecting the galaxy samples of distant clusters more than those of nearer ones thereby hampering comparisons between different clusters) is applicable to the preliminary methods used in Young \& Currie (1994) assuming poorer seeing conditions. However, the full treatment of seeing effects used by us in Young \& Currie (1995), and as outlined below, solved this potential problem as well as the hazards of slope changes also mentioned by Cellone.

Apparent differences in the shapes and slopes of the curves representing the scaling relationships can occur between clusters, or even between different datasets for the same cluster if there are both Malmquist bias and depth 
effects in one or more galaxy sample, as would appear to be the case in Cellone's Fig. 4. It is therefore important to determine the slopes at any particular point by means of a set of objects whose distances are well known (e.g. Local Group objects) or a set of objects known to be at similar distances. In either case, seeing effects as a function of projected distance must be accounted for as in e.g. Young \& Currie (1995) or Khosroshahi et al. (2000). The effect cited by Cellone as causing slope changes is one in which those galaxies smaller than a certain apparent size (relative to the size of the seeing disc) are simply excluded for distance-determination purposes because they are supposedly too severely affected by seeing ${ }^{1}$. This effect is very similar to a Malmquist bias. Curiously however, for it to produce an apparent slope change there must also be significant intra-cluster line-of-sight depth at the same timea possibility all but discounted by Cellone.

Genuine differences in the shapes and slopes of the curves must also arise because, as quantified by e.g. Young et al. (1998), the profiles of distant objects tend to be more distorted by seeing effects than those of nearer objects. Consequently the shapes of the lines defining not only the $L-n$ but also the $R-n$ and $\mu_{0}-n$ relationships are therefore themselves generally more distorted for more distant groups than they are for nearer ones.

This problem can also be countered by the procedures adopted in Young \& Currie (1995), in which we started with surface photometry of four Local Group early-type dwarfs, whose distances were already well known. Profiles of these dwarfs tend not to be affected significantly by seeing effects, simply because all of these object subtend very large solid angles on the sky on account of their proximity to us. Such profiles can then projected to greater and greater distances, taking seeing effects into account by convolving the images with normalised point-spread functions of increasing $F W H M$. The mean absolute distance to a group (or cluster of galaxies of small depth) is then the distance to which the Local Group objects have to be projected in order for their locus to match most closely that of the target group or cluster. Other methods for obtaining relative distances between groups or clusters in the absence of a Local Group locus, probably require iterative procedures in order to account for seeing differences.

\section{Intrinsic scatter}

Cellone found the scatter in his Fig. 3, an $L-n$ plot for eleven NGC 5044-Group dwarfs, to be relatively large even if the three outliers were removed. However, even the remaining eight objects differ very widely in colour $(0.67 \leq(B-V) \leq 0.97)$. This is a strong indication that their stellar populations vary greatly and that the $L-n$ relationship is therefore not applicable even to this subsample as a whole. The reasons for this have already been explained by Young \& Currie (1994, 1995 and 1998).

\footnotetext{
1 Note that such objects can be included if a full treatment of seeing effects is followed.
}

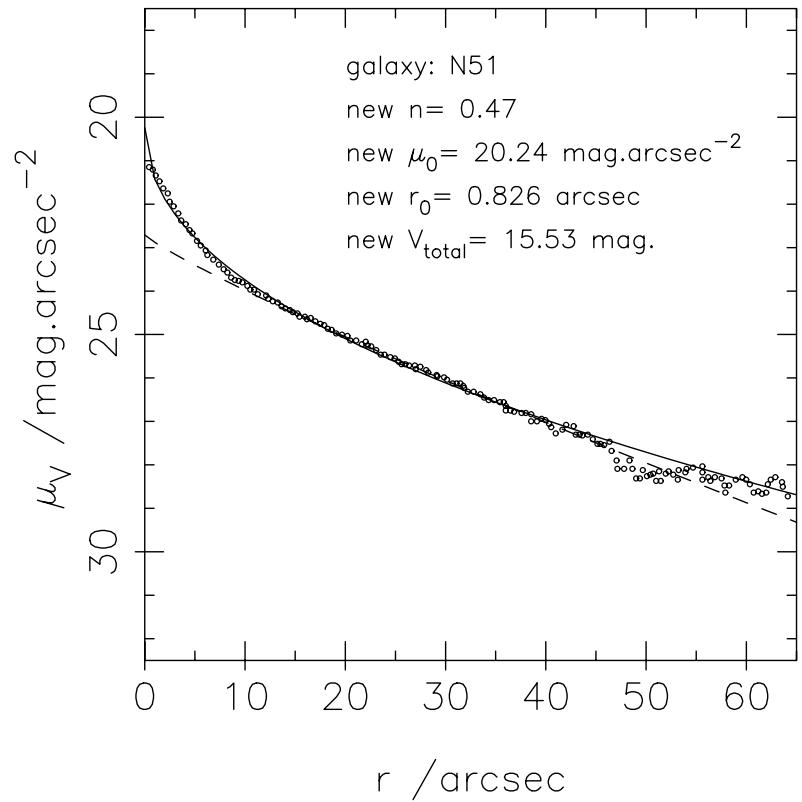

Fig. 2. Cellone's $V$-band surface-brightness profile of N51. His original fit (dashed line) and our new fit (solid line) are both shown. Unlike his fit, which excluded the galaxy's core, we fitted the whole profile excluding only $r^{*}<2$.' 0 and $r^{*}>45$." 0 . For reference, our new values for N51's Sérsic profile parameters are listed. The data points were obtained by digitising Cellone's Fig. 1

As Cellone only presented surface-brightness profiles for six (three outliers and three others) of his fifteen galaxies, it is difficult to check the degree of consistency in his profile-fitting procedures. However, from the six profiles presented in his Fig. 1, it would appear that his fitting procedures might also be responsible for increasing the observed scatter. The problems with Cellone's adopted fit for N42 have already been addressed. In the case of N51, the core of the galaxy was again ignored resulting in a very exponential fit instead of a concave one. Most worrying of all though is Cellone's fit to his profile of N95A. Although N95A clearly has a very exponential profile from visual inspection of his Fig. 1, Cellone derives an $n$ value for this galaxy of 0.17 . In order to allow readers to judge for themselves, we have re-plotted Cellone's profiles of N51 and N95A in Figs. 2 and 3 respectively. Although Cellone did not include N95A in his Fig. 3 and concedes that "its measured parameters are most probably wrong", we nevertheless have reason to be concerned about the fitting procedures adopted for the nine galaxies whose profiles were not plotted.

\section{The $R-n$ relationship}

In the absence of profile plots or data for nine of Cellone's fifteen galaxies, we are not in a position to check on the accuracy of the profile parameters he derived for the majority of his objects. However, we can compare those six objects whose profiles are featured in his Fig. 1: N30, N49 \& N50 (for which his fits seem to be reasonable and have 


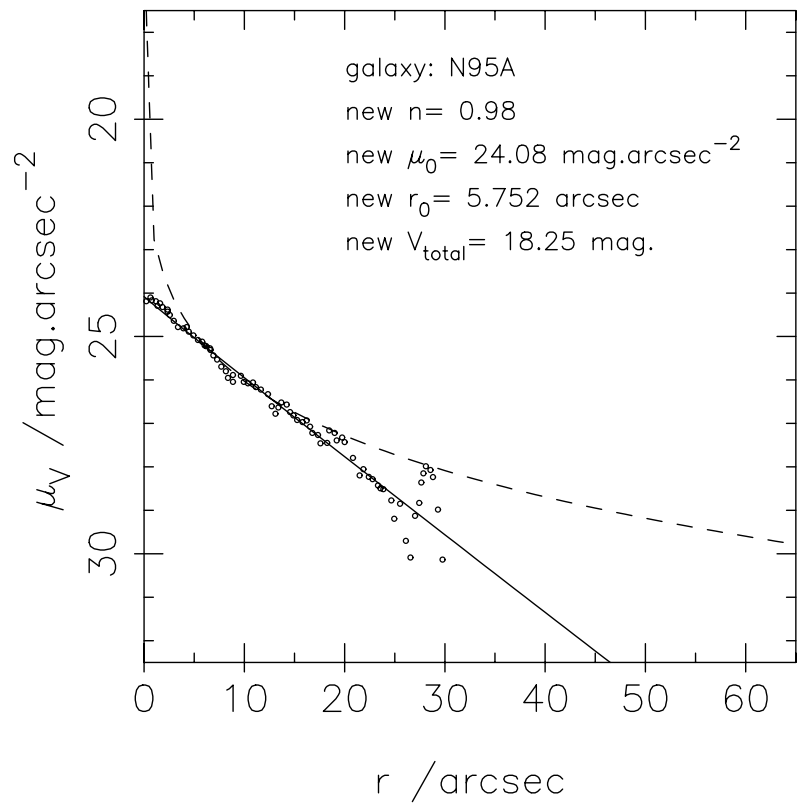

Fig. 3. Cellone's $V$-band surface-brightness profile of N95A. His original fit (dashed line) and our new fit (solid line) are both shown. We fitted the whole profile excluding only $r^{*}<$

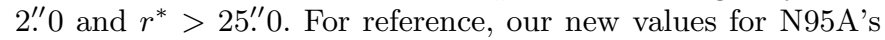
Sérsic profile parameters are listed. Note that although Cellone did not list an $r_{0}$ value for N95A, the value he derived must have been $2.1010^{-5}$ arcsec. We were able to obtain this figure from the $n, \mu_{0}$ and $V_{\text {total }}$ values that he did list. The data points were obtained by digitising Cellone's Fig. 1

been adopted here without modification), and N42, N51 \& N95A (which we re-fitted and whose new profile parameters are listed in Figs. 1-3); with the six other objects plotted in his Fig. 3: N29, N34, N66, N83, N95 \& N122 (for which profiles were not presented) and the $R$ - $n$ locus we derived for Fornax dwarfs in Sect. 6 of Young \& Currie $(1998)^{2}$.

As can be seen from Fig. 4, those six of Cellone's objects whose profile parameters could be checked (including even N95A and the irregular N49), observe a tight scalelength-shape relationship ${ }^{3}$. In fact, the observed scatter was found to be a mere 0.09 mag. Unfortunately, on account of the small sample size, it is not possible to tell whether this is due to chance or the intrinsic scatter in the $R$ - $n$ relationship really being less than 0.09 mag. This result is nevertheless very encouraging.

When all twelve objects were fitted with an $R$ - $n$ polynomial of the same form, the scatter was found to be $0.57 \mathrm{mag}$. (Though it should be possible to obtain a tighter fit with a different polynomial.) Whether this is due to differences in or problems with Cellone's profile-fitting procedures, or due to genuine scatter cannot be discerned without access to the original data. We suspect that truth probably lies somewhere in between. The locus of those ob-

\footnotetext{
2 This locus was obtained by fitting in $\log \left(r_{0}\right)-n$ space and took the form: $R=\log \left(r_{0}\right)=0.00103137 n^{-3}-0.258758 n^{-2}-$ $0.0286040 n^{-1}+1.09277$.

${ }^{3}$ We fitted a polynomial of the form $R=a n^{-2}+b n^{-1}+c$.
}

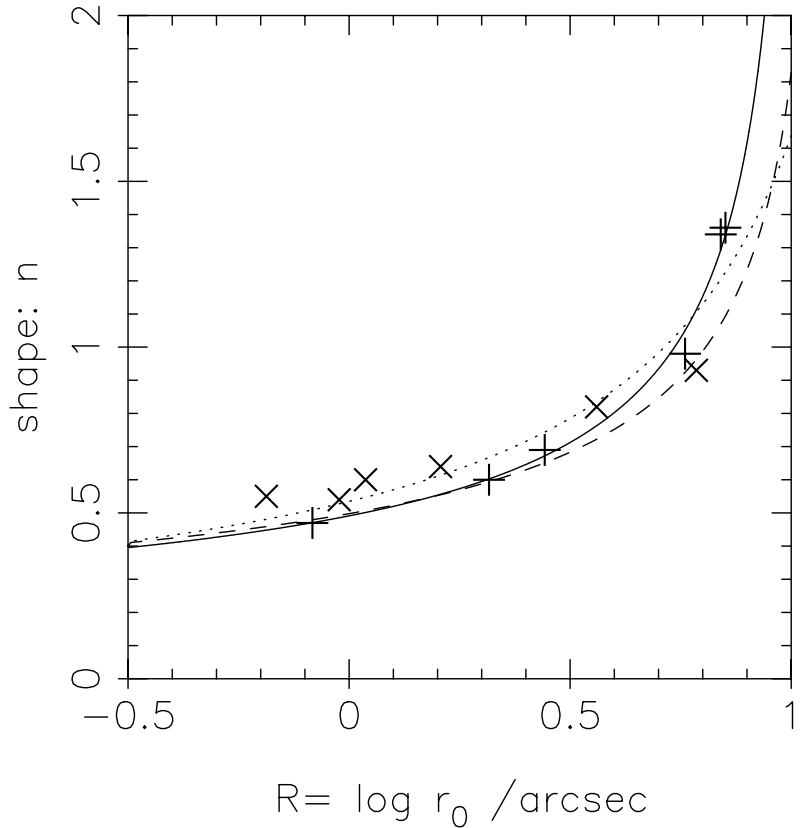

Fig. 4. Profile shape $(n)$ versus scalelength $\left(R=\log \left(r_{0}\right)\right)$ for the six NGC 5044 Group objects whose profile parameters could be checked (and re-computed in three out of six cases) ("+" symbols) and a polynomial fit to their locus (solid line). Also shown are the remaining six objects in Cellone's Fig. 3 ("X" symbols), whose profile parameters could not be checked, and a polynomial fit to all twelve objects (dotted line). The Fornax-galaxy locus from Sect. 6 of Young \& Currie (1998) is shown for reference (dashed line). Note that all fits were based on the minimisation of residuals in $R$ space

jects whose profile parameters could not be checked does appear to have a significantly different slope from that of the objects whose parameters are well known. This is evidence for some divergence in the fitting procedures.

Note that Fig. 4 cannot in itself yield an accurate relative-distance estimate for the Fornax Cluster and the NGC 5044 Group, because differences in seeing conditions (which cause slope changes) have not been taken into account. However, it is reassuring that the NGC 5044 Group appears to be slightly further away than the Fornax Cluster.

\section{Conclusions}

We have never denied that there are some practical problems in defining samples of galaxies that are directly comparable by means of the $L-n$ relationship. In fact, we discussed these issues extensively in Young \& Currie (1994, 1995 and 1998), and this is why we favoured the more general $R$ - $n$ relationship in the last two of those papers. The $R-n$ relationship is closely related to the $L-n$ one. However, it has the advantage of its plane being orthogonal to the axis defining central surface brightness (which we hypothesized was a function of the overall $M / L$ of the stellar populations present for galaxies of the same mass, physical size and profile shape). This means that 
objects of different overall $M / L$ can be compared using the $R-n$ relationship.

However, as stressed in Young \& Currie (1998), objects with colour gradients (i.e. internal variations in $M / L$ ) cannot be compared even with the latter relationship because their luminosity-profile shapes are in part the product of radial variations in $M / L$. In order to define galaxy samples by means of objective criteria and to obtain optimal results from the $R$ - $n$ relationship, it would therefore be necessary first to measure the profiles of galaxies one proposes to use in two wavebands, and then to exclude any object found to possess a significant colour gradient. We suspect that invoking a priori criteria such as the absence of significant colour gradients (in addition to more obvious ones such as the absence of giant neighbours and the exclusion of all late-type objects) would automatically lead to the exclusion of most anomalous objects such as N42. This issue will be investigated in forthcoming papers. Nevertheless, we accept that more statistical data on the goodness of fit of Sérsic's law to dwarf elliptical and dwarf spheroidal profiles are needed so that objective criteria can be determined and applied in a consistent manner by different observers. At present there are only arbitrary criteria, allowing scope for disputes on the validity of the methods. After all, Sérsic's law is only an empirical one and deviations from it can be expected. More statistics are important not only for assessing the accuracy of the distance indicators, but also for an understanding of the physics of these systems, especially if the deviations can be correlated with other factors.

Apart from the issues raised by the case of N42, we have already accounted for all but one of the other issues raised by Cellone in our previous work. His discovery of another anomalous object, N50, does however, imply that the $L-n$ relationship is not a fail-safe indicator even for individual dwarf-elliptical galaxies of $B-V \sim 0.7$; unless one takes central surface-brightness differences into account. As taking these differences into account is troublesome and in any case amounts to using the $R$ - $n$ relationship, we conclude that there is no reason to use the $L-n$ relationship as a distance indicator, if only because one can always use the more fundamental and general
$R-n$ relationship instead. For this reason, rather than for the reasons given by Cellone, we therefore find ourselves in agreement with him that the $L-n$ relationship is more useful for studying the structure of elliptical galaxies than as a distance indicator. We also very much agree with Cellone that it would be most interesting to search for more N50-like objects, to see just how common they are. Independent checks on the accuracy of his photometric profiles and colour measurements for N42 and N50 would also be worthwhile.

Finally, in this paper, we have demonstrated that Cellone's own surface-brightness profile data define a tight $R-n$ relationship for NGC 5044 Group dwarf-elliptical galaxies. This is evidence for the robustness of the $R-n$ relationship as a distance indicator.

Acknowledgements. CKY gratefully acknowledges a Chinese Academy of Sciences Senior Visiting Fellowship from Shanghai Astronomical Observatory as well as support from the K.C. Wong Education Foundation, Hong Kong, China.

\section{References}

Binggeli, B., \& Jerjen, H. 1998, A\&A, 333, 17

Biviano, A. 1997, Our best friend, the Coma Cluster (A Historical Review), in Untangling Coma Berenices: A New Vision of an Old Cluster, ed. A. Mazure, F. Casoli, F. Durret \& D. Gerbal (World Scientific, Singapore)

Cellone, S. A. 1999, A\&A, 345, 403

Durrell, P. R., McLaughlin, D. E., Harris, W. E., \& Hanes, D. A. 1996, ApJ, 463, 543

Ferguson, H. C., \& Sandage, A. 1990, AJ, 100, 1

Khosroshahi, H. G., Wadadekar, Y., Kembhavi, A., \& Mobasher, B. 2000, ApJ, 531, L103

Lucey, J. R., Currie, M. J., \& Dickens, R. J. 1986, MNRAS, 221,453

Sérsic, J. L. 1968, Atlas de Galaxias Australes, Observatorio Astronómico de Córdoba

Young, C. K., \& Currie, M. J. 1994, MNRAS, 268, L11

Young, C. K., \& Currie, M. J. 1995, MNRAS, 273, 1141

Young, C. K., \& Currie, M. J. 1998, A\&A, 333, 795

Young, C. K., Metcalfe, N., Zhu, J., Wu, H., \& Chen, J. S. 1998, A\&AS, 130, 173 